Yolumen 4, nímerol: 1991.

http://dx.doi.org/10.5209/rev_REMA.1991.v4.n1.18007

\title{
On a Critical Threshold for Nonlinear Diffusion Equations
}

\author{
M. CHIPOT and R. KFRSNER
}

ABSTRACT. We study some aspects of the asymptotic behavior of the solutions to a class of nonlinear parabolic equations.

\section{INTRODUCTION}

Set $\mathbf{R}_{+}=\{t \in \mathbf{R} \mid t>0\}$

We are concerned here with weak solutions to nonlinear parabolic equations of the type

$$
\begin{gathered}
u_{t}=u(u)_{x x}+f(u)_{x} \quad \text { in } \quad \mathbf{R} \times \mathbf{R}_{+} \\
u(x, 0)=u_{0}(x) \geq 0 \quad \text { in } \quad \mathbf{R} .
\end{gathered}
$$

Without loss of generality we can assume $a(0)=0, f(0)=0$. (Indeed one can replace in (1) $a$ by $a-a(0)$ and $f$ by $f-f(0)$ ).

If for some $\tau>0$ we have

$$
f(\tau)=0
$$

we would like to show that, under additional assumptions on $f$. some aspects of the asymptotic behavior of $u(x, t)$ when $t \rightarrow+\infty$ are governed, roughly speaking, by the position of $u_{0}(x)$ with respect to $\tau$.

It is interesting to compare our results with the results for Fisher type equations (see [A], [K2]).

1980 Mathematics Subject Classification (1985 revision): 35 B 40, 35 G 30. 35 . I 70, 35 K 55. Editorial de la Universidad Complutense. Madrid. 1991. 
The paper is divided as follows. In section 2 we discuss the existence of some stationary solutions to (1). In section 3 we state and prove our main theorems. At last, in section 4 we give some examples of applications and discuss briefly the possible extensions of our results in higher dimensions.

\section{EXISTENCE OF STATIONARY SOLUTIONS}

Our method relies strongly on the existence of stationary solutions to the equation (1). We will assume here that.

$a:[0,+\infty) \rightarrow[0,+\infty), a(0)=0, a(u)$ smooth, increasing for $u>0$.

We will denote by $a^{-1}$ the inverse function of $a$. Moreover, we will suppose that:

$$
f:[0,+\infty) \rightarrow \mathbf{R}, f(0)=0, f \text { of class } C^{1}
$$

Let $\tau$ be a strictly positive constant. The case of interest here is when (3) holds and

$$
\begin{gathered}
f(u)<0=f(\tau) \quad \forall u \in(0, \tau) \\
-\infty<f^{\prime}(0)<0 .
\end{gathered}
$$

Since our data are smooth and

$$
h(s)=-f\left(a^{-1}(a(\tau)-s)\right)
$$

is such that $h(0)=0$ one has for $s>0$ close to 0 :

$$
h(s)=O(s)
$$

and thus

$$
-\int^{(s t s)^{-}} \frac{d s}{f\left(a^{-1}(s)\right)}=+\infty
$$

We will consider the case where the problem (1) is degenerate and the case where it is not $-i, e$. the two cases (see $[\mathrm{K} 1],[\mathrm{P}]$ ):

$$
\int_{0+} \frac{1}{a^{-1}(v)} d v<+\infty
$$


and

$$
\int_{0+} \frac{1}{a^{-1}(v)} d v=+\infty
$$

Under the above assumptions we can prove:

Proposition 1: Assume (3),-,(7), then

(i) if (9) holds, there exists one and only one stationary solution $s(x)$ to (I) such that

$$
\begin{gathered}
s(x)=0 \quad \forall x \leq 0, \\
\text { sincreasing on }(0,+\infty) . \\
\lim _{x \rightarrow+\infty} s(x)=\tau .
\end{gathered}
$$

(ii) If (10) holds, (1) has no stationary solution s such that $s(x)=0$ for $x \leq 0, s(x)>0$ for $x>0$. For any $c \in(0, \tau)$, there exists one and only one stationary solution $s(x, c)$ to (I) such that

$$
\begin{gathered}
s(0, c)=c . \\
s(x, c) \text { increasing on }(-\infty,+\infty) . \\
\lim _{x \rightarrow+\infty} s(x, c)=\tau, \quad \lim _{x \rightarrow-\infty} s(x, c)=0 .
\end{gathered}
$$

Proof: A stationary solution to (1) has to satisfy

$$
a(u)_{x x}+f(u)_{x}=0 \text { in } \mathbf{R} \leqq a(u)_{x}+f(u)=C s t . \text { in } \mathbf{R} \text {. }
$$

If one wants to have $u(x) \rightarrow \tau$ when $x \rightarrow+\infty$ we must have, Cst. $=0$. So, we look for $u$ solution to

$$
a(u)_{x}+f(u)=0 \text { in } \mathbf{R}
$$

Assume first that we are in case (i).

For $s \in[0, a(\tau))$ define

$$
F(s)=-\int_{11}^{s} \frac{d s}{f\left(a^{-1}(s)\right)}
$$


First remark that by (7). one has

$$
\frac{1}{f^{\prime}\left(a^{-1}(s)\right)}=\frac{a^{-1}(s)}{a^{-1}(s) f\left(a^{-1}(s)\right)} \sim \frac{1}{f^{\prime}(0) a^{-1}(s)}
$$

when $s \rightarrow 0$ and thus by (9). (18) makes sense. Moreover. by (6). (8), $F$ is an increasing function from $[0, a(\tau))$ onto $[0,+\infty)$. Denote by $F^{-1}$ its inverse and set

$$
s(x)=0 \quad \forall x \leq 0 \quad, \quad s(x)=a^{-1}\left(F^{-1}(x)\right) \quad \forall x>0
$$

Clearly satisfies (11), (12). (13). Moreover one has

$$
a(s(x))=0 \quad \forall x \leq 0, \quad a(s(x))=F^{-1}(x) \quad \forall x>0
$$

and this function has no jump on $\mathbf{R}$. So, in the distributionnal sense one has

$$
a(s(x))_{x}=F^{-1}(x)_{x}=-f(s(x))
$$

and $s$ is the stationary solution we are looking for (see (17)).

Assume now that we are in case (ii).

If $s$ is a stationary solution to (1) such that that

$$
s(x)=0 \quad \forall x \leq 0 \quad . \quad s(x)>0 \quad \forall x>0
$$

then $v=a(s(x))$ is a solution to

$$
v_{x}=-f\left(a^{-1}(v)\right)
$$

such that

$v(x)=0 \quad \forall x \leq 0, v(x)>0 \quad \forall x>0, v$ locally increasing for $x>0$

This is not possible. Indeed, for "positive one would have

$$
\frac{v_{x}(x)}{a^{-1}(v(x))}=-\frac{\left.f\left(a^{-1}(v(x))\right)\right)}{a^{-1}(v(x))}
$$

and thus for $s>0, r>0$ small enough

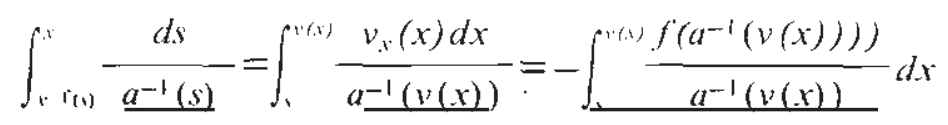


and a contradiction (by (10), (7)) when $s \rightarrow 0$.

Now, if we select $c \in(0, \tau)$ then we can define

$$
F(s)=-\int_{u(u)} \frac{d s}{f\left(a^{-1}(s)\right)} .
$$

Clearly, $F$ is a one-to-one increasing map from $(0, a(\tau))$ into $(-\infty,+\infty)$ so that

$$
s(x, c)=a^{-1}\left(F^{-1}(x)\right)
$$

is the stationary solution we are looking for.

Remark 1: In the case where $f$ is not differentiable the above analysis could have been made in terms of the Osgood condition. i.e. in considering the cases

$$
\int_{0+}-\frac{1}{f\left(a^{-1}(v)\right)} d v<+\infty \quad \int_{0+}-\frac{1}{f\left(a^{-1}(v)\right)} d v=+\infty
$$

However, the assumption (7) is useful in what follows and we did prefer state Proposition 1 under this form.

\section{THE MAIN RESULTS}

Let us consider now $u=u(x, t)$ the weak solution to (1), (2). It is well known (see [DK], [G]) that under suitable assumptions on $a$, $f$, such an equation has a unique weak solution.

We have:

Theorem 1: Assume that (3),-.(7) hold. Moreover, assume that

$$
\frac{f(u)}{l} \text { increasing on }(\tau, \tau+\delta)
$$

for some $\delta>0$.

Then:

(i) If (9) holds and

$$
\liminf _{i \rightarrow \infty} u_{0}(x)>\tau
$$


(ii) if (10) holds and if

$$
u_{0}(x) \geq \alpha>0 \quad \forall x, \quad \liminf _{x \rightarrow+\infty} u_{0}(x)>\tau
$$

for any $x \in \mathbf{R}$ one has

$$
\liminf _{t \rightarrow x^{+}} u(x, t)>\tau
$$

Proof: By (19) if $\epsilon$ is small enough there exists a unique $\tau_{t}>\tau$ such that

$$
\frac{f\left(\tau_{t}\right)}{\tau_{t}}=\epsilon, \frac{f(u)}{u}<\epsilon \text { on }\left(0, \tau_{t}\right)
$$

If we set

$$
f_{e}(u)=f(u)-\epsilon u
$$

then $f_{\mathrm{t}}$ satisfies (3), (5), (6), (7) with $\tau=\tau_{f}$. If we apply Proposition 1, we can denote by $s_{*}(x)$ and $s_{t}(x, c)$ the solutions to

$$
a(u)_{x x}+(f(u)-\epsilon u)_{x}=0 \text { in } \mathbf{R}
$$

corresponding to cases (9) and (10) and satisfying respectively (11)-(13), (14)(16) with $\tau=\tau_{\epsilon}$. Choosing $\epsilon$ small enough one can clearly have:

$$
\tau<\tau_{e}<\liminf _{x \rightarrow+\infty} u_{0}(x)
$$

and thus, for $x$ large enough, i.e. $x \geq M$, we have

$$
u_{0}(x)>\tau_{\varepsilon}
$$

Then, in case (i) one has

$$
u_{0}(x) \geq s_{\mathrm{t}}(x-M)
$$

and in case (ii)

$$
u_{0}(x) \geq s_{t}(x-M, \alpha)
$$

(see (21)). Now, by (23), remark that

$$
s_{\epsilon}(\epsilon t+x-M) \text { and } s_{t}(\epsilon t+x-M, \alpha)
$$


are solutions to (1). Since by (24), (25), $u_{0}$ is larger than these solutions at time $t=0$, we deduce from the maximum principle that

$$
u(x, t) \geq\left\{\begin{array}{l}
s_{t}(\epsilon t+x-M) \\
o r \\
s_{t}(\epsilon t+x-M, \alpha) .
\end{array}\right.
$$

Letting $t \rightarrow+\infty$ we get by (13), (16)

$$
\liminf _{t \rightarrow+\infty} u(x, v) \geq \tau_{t}>\tau
$$

This ends the proof.

Remark 2: It is clear from the proof that in this theorem one can allow $f^{\prime}$ to vanish at 0 i.e $-\infty<f^{\prime}(0) \leq 0$.

We can also prove:

Theorem 2: Assume that (3),-, (7) hold. Moreover, assume that

$$
f \text { is increasing on }(\tau-\delta, \tau)
$$

for some $\delta>0$.

$$
0 \leq u_{0}(x) \leq \tau^{\prime}<\tau \forall x, u_{0}(x) \equiv 0 \quad \forall x \leq Z
$$

where $Z$ is some constant. Then

(i) if (9) holds one has for every $x$ :

$$
u(x, t) \equiv 0 \text { for t large enough. }
$$

(ii) If (I0) holds one has for every $x$

$$
\lim _{x \rightarrow+\infty} u(x, t)=0
$$

Proof: By (26) if $\epsilon$ is small enough, there exists $\tau_{\epsilon}$ such that

$$
\begin{gathered}
\tau^{\prime}<\tau_{\epsilon}<\tau \\
f(u)+\epsilon u<0 \quad \text { on } \quad\left(0, \tau_{\epsilon}\right), f\left(\tau_{\epsilon}\right)+\epsilon \tau_{\epsilon}=0 .
\end{gathered}
$$


If we set

$$
f_{f}(u)=f(u)+\epsilon u
$$

then $f_{t}$ satisfies (3), (5), (6), (7) with $\tau=\tau_{t}$. If we apply Proposition I, we can denote by $x_{-t}(x)$ and $s_{-t}(x, c)$ the solutions to

$$
a(u)_{x x}+(f(u)+\epsilon u)_{x}=0 \quad \text { in } \quad \mathbf{R}
$$

corresponding to cases (9) and (10) and satisfying respectively (11)-(13). (14)(16) with $\tau=\tau_{\mathrm{E}}$. If we choose $M$ large enough then we have in case (i) or (ii):

$$
\begin{gathered}
s_{-s}(x+M) \\
o r \geq u_{0}(x) \\
s_{-s}(x+M, r) .
\end{gathered}
$$

Hence, by the maximum principle (see the preceding proposition, note that, by $(27), s_{-+}(-t+x+M)$ and $s_{-t}(-t+x+M, c)$ satisfy (1)):

$$
\begin{gathered}
s_{-\varepsilon}(-\epsilon t+x+M) \\
o r \geq u(x, t) \\
s_{-\varepsilon}(-\epsilon t+x+M, c) .
\end{gathered}
$$

In case (i) from the above inequality we get

$$
u \equiv 0
$$

as soon as $-\epsilon t+x+M \leq 0$. In case (ii) the result follows by letting $1 \rightarrow+\infty$.

Remark 3: In the case (i). we have proved in fact that if $u_{0}$ has its support bounded below then, so does $u(., t)$ for any $t$. Moreover, the left front of the support is moving to the right with a velocity greater or equal to $\epsilon$.

The situation can be completely different if in (7) we allow the value $f^{\prime}(0)=0$. Indeed. let us assume that we have for $u$ small, non negative

$$
\begin{gathered}
\left|f^{\prime}(u)\right| \leq C \cdot u^{\beta} \\
a^{\prime}(0)=0, \quad\left|a^{\prime \prime}(u)\right| \leq C \cdot u^{\delta}
\end{gathered}
$$


where $C, \beta$ are positive constants and $\delta>-1$.

Then, we can prove:

Theorem 3: Assume that (4). (5), (28), (29) hold. Then if

$$
u_{0}\left(x_{0}\right)>0
$$

we have

$$
u\left(x_{0}, t\right)>0 \quad \forall t>0
$$

Proof: Changing $x$ in $x+x_{0}$ in what follows we can assume without loss of generally that $x_{0}=0$. Consider

$$
v=v(x, t)=e^{-n x}\left(\left[\epsilon-x^{2} e^{i}\right]^{+}\right)^{n}
$$

where $\epsilon, W$ are positive constants that will be chosen later on and []$^{+}$ denotes the positive part of a function.

Set $A=\epsilon-x^{2} e^{t}$. In the domain

$$
H=\left\{(x, t) \mid \epsilon-x^{2} e^{\prime}>0\right\}
$$

we have:

$$
\begin{aligned}
& v_{t}=-w^{\prime} A^{w} e^{-w r}-x^{2} w \cdot A^{w-1} e^{-t(w-1)} \\
& v_{x}=-2 x w \cdot A^{w-1} e^{-1(w-1)} \\
& v_{x w}=-2 w \cdot A^{w-1} e^{-1(w-1)}+4 x^{2} w(w-1) A^{w-2} e^{-t(w-2)} .
\end{aligned}
$$

Set

$$
\begin{aligned}
L v & =v_{1}-a(v)_{x x}-f(v)_{x} \\
& =v_{1}-a^{\prime \prime}(v)\left(v_{x}\right)^{2}-a^{\prime}(v) v_{x, x}-f^{\prime \prime}(v) v_{x}
\end{aligned}
$$

We obtain

$$
L v=-w A^{\prime \prime} e^{-n+1}-x^{2} w^{\prime} A^{n-1} e^{-1(n-1)}-a^{\prime \prime}(v) 4 x^{2} w^{2} A^{2(w-1)} e^{-2 l(w-1)}+
$$

$a^{\prime}(v) 2 w A^{\prime \prime-1} e^{-f(w-1)}-a^{\prime}(v) 4 x^{2} w(w-1) A^{w-2} e^{-t(w-2)}+f^{\prime}(v) 2 x w A^{w-1} e^{-t(w-1)}$. 
By (29) we have for $v>0, v$ small

$$
\begin{aligned}
& \left|a^{\prime}(v)\right| \leq\left|\int_{0}^{v} a^{\prime \prime}(s) d s\right| \leq \int_{0}^{\prime \prime}\left|a^{\prime \prime}(s)\right| d s \\
& \leq \int_{0}^{r} s^{s} d s=\frac{C}{1+\delta} v^{\cdot 1+\delta}=C^{\prime} v^{\gamma}
\end{aligned}
$$

where we have set

$$
C^{\prime}=\frac{C}{1+\delta}, \gamma=1+\delta>0 .
$$

Combining this, (28) and the inequality

$$
|2 x| \leq x^{2}+1
$$

we obtain for $\epsilon$ small enough (note that $0 \leq v \leq \epsilon^{\text {") }}$ )

$$
\begin{aligned}
& L v \leq-w A^{n} e^{-w+}-x^{2} w A^{w-1} e^{-(w-1)}+4 C x^{2} w^{2} A^{w}+2(w-1) e^{-1(w b+2 w-2)} \\
& +2 C^{\prime} w A^{n^{\prime} \gamma+w^{\prime}-1} e^{-1(w \gamma+w-1)}+4 C^{\prime} x^{2} w(w-1) A^{w \gamma+w-2} e^{-1(w \gamma+n-2)} \text {. } \\
& +C x^{2} w \cdot A \cdot \beta+w-1 e^{-1(w \beta+n-1)}+C w \cdot A \cdots \beta+w-1 e^{-1(w \beta+w-1)} \text {. }
\end{aligned}
$$

Hence:

$$
\begin{aligned}
& L v \leq-w A^{w} e^{-w \gamma}\left\{1-2 C^{\prime} A^{w+\gamma-1} e^{-1(w \gamma-1)}-C A^{n / \beta-1} e^{-1(w \beta-1)}\right\} \\
& -x^{2} w A^{w-1} e^{-1(w-1)}\left\{1-4 C w A^{w \delta+n-1} e^{-1(n \delta+w-1)}\right. \\
& \left.-4 C^{\prime}\left(w^{\prime}-1\right) \cdot A^{w \gamma-1} e^{-(n \gamma-1)}-C A^{w \beta} e^{-n \beta n}\right\} .
\end{aligned}
$$

Select now w' such that

$$
w \gamma>1, w \beta>1, w>1
$$

We have:

$$
\begin{gathered}
1-2 C^{\prime} A^{w \gamma-1} e^{-r(w \gamma-1)}-C A^{w \beta-1} e^{-(w \beta-1)} \geq 1-2 C^{\gamma} A^{w \gamma-1}-C A^{w \beta-1}>0 \\
1-4 C w A^{w \delta+w-1} e^{-6(n \delta+w-1)}-4 C^{\prime}(w-1) A^{w \gamma-1} e^{-1(w \gamma-1)}-C A^{w / \beta} e^{-w \beta r} \geq \\
1-4 C w A^{w \delta+w-1}-4 C^{\prime}(w-1) A^{w \gamma-1}-C A^{w \beta}>0
\end{gathered}
$$


provided that $\epsilon$ is small enough (see (32), recall that $A \leq \epsilon$ ). Now, if we take into account the fact that $a^{\prime}, f^{*}$, vanishe at 0 we obtain in the distributional sense in $\mathbf{R} \times \mathbf{R}_{+}$:

$$
L v \leq 0
$$

for $\epsilon$ small enough. Now from (30), i.e. $u(0)>0$, we have for $\epsilon$ small enough

$$
v(x, 0) \leq u_{0}(x) \quad \forall x \in \mathbf{R} .
$$

Hence, by the maximum principle

$$
v(x, t) \leq u(x, t) \quad \text { in } \mathbf{R} \times \mathbf{R}_{+}
$$

and in particular

$$
0<v(0, t) \leq u(0, t) \quad \forall t \in \mathbf{R}_{+} .
$$

This concludes.

Remark 4: Note that in this theorem we did not use the assumption (6).

Remark 5: A consequence of Theorem 3 is, under the same assumptions, that when $u\left(x_{0}, t_{0}\right)>0$ then $u\left(x_{0}, t\right)>0 \forall t>t_{0}$. Thus, this allows the definition of the free boundary as a function $x=s(t)$. (By (29), (31) it is easy to show that we are in the degenerate case). Moreover, $t=r(x)$ is a monotome graph (see $[\mathrm{G}]$ ).

\section{EXAMPLES}

Let us consider for instance :he equation

$$
u_{t}=\left(u^{\prime \prime}\right)_{x x}+\left(u^{\prime \prime}\right)_{x}-\left(u^{\prime \prime}\right)_{x}
$$

where the real numbers $m, n, p$ satisfy $m \geq 1, n \geq 1, p \geq 1$. The equation is of the type of equation (1) if we set

$$
f(u)=u^{n}-u^{p} .
$$

If $n>p$ then (6) holds with $\tau=1$ (see (3)). Moreover, (19) and (26) hold. Indeed, $f(u)=u^{n}-u^{r}$ is increasing on the left of I since

$$
f^{\prime}(1)=n-p>0
$$


and similarly for $\frac{f(u)}{u}=u^{\prime \prime-1}-u^{\prime \prime-1}$. Thus, in this case Theorem I holds (see Remark 1).

If now we suppose $p=1 . n>1$ then

$$
f(u)=u^{n}-u \quad \cdot \quad f^{\prime}(0)=-1
$$

and Theorem 2 holds. In any other cases we are in situation to apply Theorem 3 provided that (28) holds (sec Remark 3).

Let us examine some particular cases.

4.1- Case $m=1, p=1, n=2$.

The problem is not degenerate and, as we just saw. the theorems 1 and 2 apply. Now the stationary solutions can be computed explicitely. Indeed, it is easy to see that for $\in \in(0.1)$ the equation

$$
u_{r}=u_{x x}+\left(u^{2}-u \pm \epsilon u\right)_{x}
$$

admits as stationary solutions (see the notation in Theorem 1 or 2)

$$
s_{ \pm+}(x, c)=(1 \pm \epsilon)\left\{\frac{1}{1+\left(\frac{(1 \pm t)}{i}-1\right) e^{-(1 \pm t) x}}\right\}
$$

Clearly when $x \rightarrow+\infty$

$$
s_{t}(x, c) \rightarrow 1+\epsilon
$$

and

$$
r_{-+}(x, c) \rightarrow 0
$$

when $x \rightarrow-\infty$. This makes explicit the asymptotic behavior described in Theorem 1 and Theorem 2, the solution of the time dependent problem being given by

$$
s_{\text {. } e t}( \pm t+x \pm M) \text {. }
$$

$4.2-$ Case $m=n>1, p=1$

The equation (33) is now degenerate but Theorems 1 and 2 both apply. It is easy to see that, when $\epsilon \in(0,1)$, the stationary solutions to

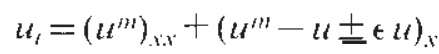


are given by (see proposition 1)

$$
s_{ \pm e}(x)=(1 \pm \epsilon)^{\prime \prime \prime-1}\left\{\left[1-e^{-m-1}{ }^{m}\right]^{+}\right\}^{m-1}
$$

where [ $]^{+}$denotes the positive part of the function into brackets. Clearly when $x \rightarrow+\infty$

$$
s_{r}(x) \rightarrow(1+\epsilon)^{m-1}
$$

which gives the explicit asymptotic behavior of $s_{\mathrm{t}}$. Considering

$$
s_{-t}(-\epsilon t+x+M)
$$

allows us to see how the back front is moving.

4.3 - Case $m>1, n=2 m-1, p=1$.

The equation (33) is again degenerate. Theorems 1 and 2 both apply. It is easy to see that, when $\epsilon \in(0,1)$, the stationary solutions to

$$
u_{i}=\left(u^{m}\right)_{x x}+\left(u^{n}-u \pm \epsilon u\right)_{x}
$$

are given by

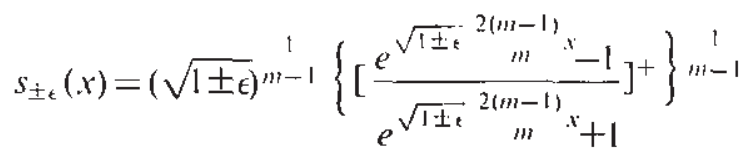

The same analysis than in case 4.2 holds in this case.

In higher dimensions it is clear that our techniques allow to get some information on the asymptotic behavior of the solution to parabolic equations of the type

$$
\begin{gathered}
u_{t}=\Delta a(u)+f(u)_{\xi} \text { in } \quad \mathbf{R}^{\prime \prime} \times \mathbf{R}_{+} \\
u(x, 0)=u_{0}(x) \geq 0 \text { in } \mathbf{R}^{n} .
\end{gathered}
$$

Here $\Delta$ is the usual Laplace operator and $f(u)_{\xi}$ denotes the derivative of $f(u)$ in the direction of the unit vector $\xi$.

Indeed, it is enough to remark that if $s$ is a stationary solution in $\mathbf{R}$ to

$$
u_{i}=a(u)_{\xi \xi}+f(u)_{\xi}
$$


then $s$ is also a stationary solution to (34) in $\mathbf{R}^{n}$ and thus comparison can be made and results similar to the ones in one dimension obtained.

Acknowledgements: Part of this paper was written when the second author was visiting the University of Metz. We would like to thank this institution for its support. We thank also J. 1. Diaz for valuable comments during the final stage of the preparation of this work.

\section{References}

[A] D. G. Aronson: Density dependent interaction-diffusion sywems. Proceedings of Advanced Seminar on Dynamics and Modelling of reactive systems, Acad. Press, New York, 1980.

[DK] J. I. DIA\%, R. KI:RSNER: On a nonlinear degenerate parabolic equation in infiltration or evaporation through a porous medium. J. Differential equations 69 (1987), p. 368-403.

[G] B. H. Gubris: Improved theory for a nonlinear degenerate parabolic equationt. Ann. Scuola Norm. Sup. Pisa, Cl. Sci. (4), Vol. XVI, Fasc. 2, (1989), p. 165-224.

[K1] A. S. KAI_ASHNiKov: On the equations of the type of non stationary filtration with infinite velocity of propagation of perturbances. Vestnik Moskov. Univ. Ser. Math. Mech. (6), 1972, p. 45-49.

[K2] A. S. Kal.Ashnikov: Some problems of the qualitative theory of nonlinear degenerate second order parabolic equations. Russian Math. Surveys 42 (1987), p. 169-222,

[P] L. A. Priletier: On the existence of an interface in nonlinear diffusion processes. Lecture Notes in Math. 415, Springer Verlag (1974), Berlin.

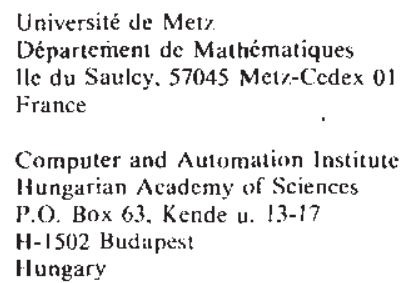

